\title{
Texturing Industrial Multicrystalline Silicon Solar Cells
}

\author{
D. Macdonald, A. Cuevas, M. Kerr, C. Samundsett, D. Ruby ${ }^{1}$, S. Winderbaum ${ }^{2}$ and A. Leo ${ }^{2}$ \\ Centre for Sustainable Energy Systems, Dept. of Engineering, FEIT, \\ Australian National University, Acton, ACT 0200, AUSTRALIA \\ ${ }^{1}$ Sandia National Laboratories, Albuquerque, NM, USA \\ ${ }^{2}$ BPSolar, Homebush Bay, AUSTRALIA \\ E-mail: daniel@faceng.anu.edu.au
}

\begin{abstract}
Three potential techniques for texturing commercial multicrystalline silicon solar cells are compared on the basis of reflectance measurements. Wet acidic texturing, which would be the least costly to implement, produces a modest improvement in reflection before antirflection coating and encapsulation, whereas maskless reactiveion etching texturing, and especially masked reactive-ion etched 'pyramids', generate a larger gain in absorption. After antireflection coating and encapsulation however, the differences between the methods are reduced. Short-circuit current measurements on wet acidic textured cells reveal that there is a significant additional current gain above that expected from the reduced reflection. This is attributed to both light-trapping and oblique coupling of incident light into the cell, resulting in generation closer to the junction.
\end{abstract}

\section{INTRODUCTION}

Multicrystalline silicon (mc-Si) solar cells currently account for around 50\% of worldwide PV production, and their share of the market is steadily increasing. In general however, commercial mc-Si cells have lower efficiencies than their single-crystal counterparts. One of the main reasons for this difference is the lack of a costeffective method of texturing mc-Si, a process that is straightforward in single-crystal cells.

Texturing the front surface of a solar cell generally results in improved performance, mostly due to an increase in the short-circuit current. This increase arises from three distinct mechanisms, all of which are related to the fact that the incident photons strike the cell surface at an angle.

Firstly, some light rays will be reflected from one angled surface merely to strike another, resulting in an improved probability of absorption, and therefore reduced reflection. Secondly, photons refracted into the silicon will propagate at an angle, causing them to be absorbed closer to the junction than would occur with a planar surface. This is especially relevant in material with diffusion lengths comparable to or less than the cell thickness, such as many multicrystalline silicon wafers. Thirdly, long-wavelength photons which are reflected from the rear surface back to the front will encounter an angled silicon surface, improving the chance of being internally reflected, either at the silicon interface or at the glass surface, and providing another chance for absorption. This final process is referred to as light-trapping, and gives an improved response to infra-red light.

Single-crystal silicon solar cells are generally textured with random pyramids, which are produced by etching in an alkaline solution such as $\mathrm{KOH}$ or $\mathrm{NaOH}$. This is made possible by the anisotropic (i.e. orientation dependent) nature of these etching solutions, in conjunction with an appropriate choice of the crystal plane orientation at the wafer surface. The random nature of the crystal orientation of multicrystalline silicon wafers makes such techniques much less effective for this material because only a handful of grains are properly orientated. In addition, alkaline solutions can cause unwanted steps and crevasses between the grains. In this work, we examine and compare some of the texturing methods that appear to have potential as cost-effective processes. Specifically, wet acidic texturing and masked and maskless Reactive Ion Etching (RIE) processes are studied.

\section{TEXTURING TECHNIQUES FOR MULTICRYSTALLINE SILICON}

Numerous techniques for texturing mc-Si silicon have been examined in the past. Some have focussed on the use of RIE, either in conjunction with a mask to achieve large, regular features (Winderbaum et al, 1997), or without a mask to produce much smaller and more random texture (Ruby et al, 1999). Other approaches have been based on isotropic wet acidic etching (De Wolf et al, 2000). All of these methods produce lower reflection than can be 
achieved with the standard alkaline damage-removal etch used today in industry.

Wet acidic texturing, performed with solutions containing $\mathrm{HF} / \mathrm{HNO}_{3}$ which tend to etch isotropically, can result in features with rounded surfaces, as opposed to flat-sided features which arise from anisotropic etches (de Wolf et al, 2000). These rounded features produce a scalloped surface that can have good reflection properties. An important issue with this technique is that the feature growth need to be 'seeded' in some way, otherwise a planar surface will result. This seeding can be provided by surface damage caused during wafer sawing (maskless acidic etching), or, in more complex methods, by defined holes through protective layers such as oxides, nitrides or polymers (masked acidic etching). In this paper we examine and evaluate a maskless acidic etching technique. Such a technique does not provide the extremely low reflection of some other methods, but does have the advantages of being relatively easy to implement and low-cost.

Reactive Ion Etching (RIE) is a method for 'dry' etching silicon without the use of large quantities of liquid solutions. It is based on placing wafers in a plasma of highly reactive ions. Under certain conditions, the etching dynamics produce deep surface features that have excellent reflection properties. Like wet acidic etching, RIE can be performed in a maskless fashion that produces somewhat randomly placed features, or in conjunction with a masking layer to produce more even features. In this paper, we compare the wet acidic texturing with both a masked and a maskless RIE texturing method. The maskless RIE texturing was performed at Sandia laboratories in the USA using a proprietary 'metal-assisted' process (Ruby et al, 1999). Masked RIE texturing was created by etching through phtolithographically-defined holes in a metal layer which is subsequently removed (Winderbaum et al, 1997). Both of these techniques offer promise for very low reflectance surfaces with minimal chemical waste, an issue that will become increasingly important in the photovoltaic industry. However, they are likely to be more costly to implement than wet chemical methods.

\section{RESULTS AND DISCUSSION}

To provide a baseline with which to compare the three types of texture studied in this paper, we have characterised wafers from BPSolar's current industrial alkaline-etching method for producing a damage-free, but optically flat, surface. We have also studied as-cut wafers, which remain in the damaged state after wafer sawing. Because of the surface damage, they have relatively low reflection. However, the damage also produces many regions of high recombination, which severely degrade cell performance. This highlights the important point that generating good texture has to be coupled with a relatively damage-free surface.

\subsection{Electron Micrographs of As-Cut, Etched and Textured Wafers}

Figure 1 shows Scanning Electron Micrographs (SEMs) of multicrystalline silicon wafers with various surfaces. In the as-cut wafer (top two images), the heavy surface damage is evident as deep fissures and cracks, resulting in reasonable reflection control but high recombination. The next two images are of a standard industrial alkalineetched wafer. It appears to have some texturing, but this is both shallow and highly grain-dependent, and when averaged over many grains is almost as reflective as a perfectly flat wafer. This reflects the fact that the alkaline etching solution used by BPSolar is relatively isotropic in comparison to solutions sometimes used for texturing single-crystal wafers.

The following two images are of an acidic textured wafer, which was performed with a $\mathrm{HF} / \mathrm{HNO}_{3}$ solution. A wetting agent was added to produce more uniform texturing. Approximately 5-10 $\mu \mathrm{m}$ of silicon was removed from each surface. Although the surface damage is removed, its original presence is nevertheless critical, since it acts as a seeding layer for the texturing. The resulting surface contains smooth sided, bowl-like features.

Figure 1 also shows images of the maskless RIE texturing performed at Sandia. These wafers have somewhat randomly-placed but nevertheless deep features with steep sides, which provide very low reflection. Note that the features of these RIE textured wafers are much smaller than those of the acidic textured sample (the two images on the right have the same magnification, 5000x). Finally, a masked RIE textured wafer is also shown at the bottom of Figure 1. These features are very regular and steep-sided, with a distance of $7 \mu \mathrm{m}$ between pyramid peaks. The pyramids are approximately the same size as the features on the wet acidic textured wafer. 

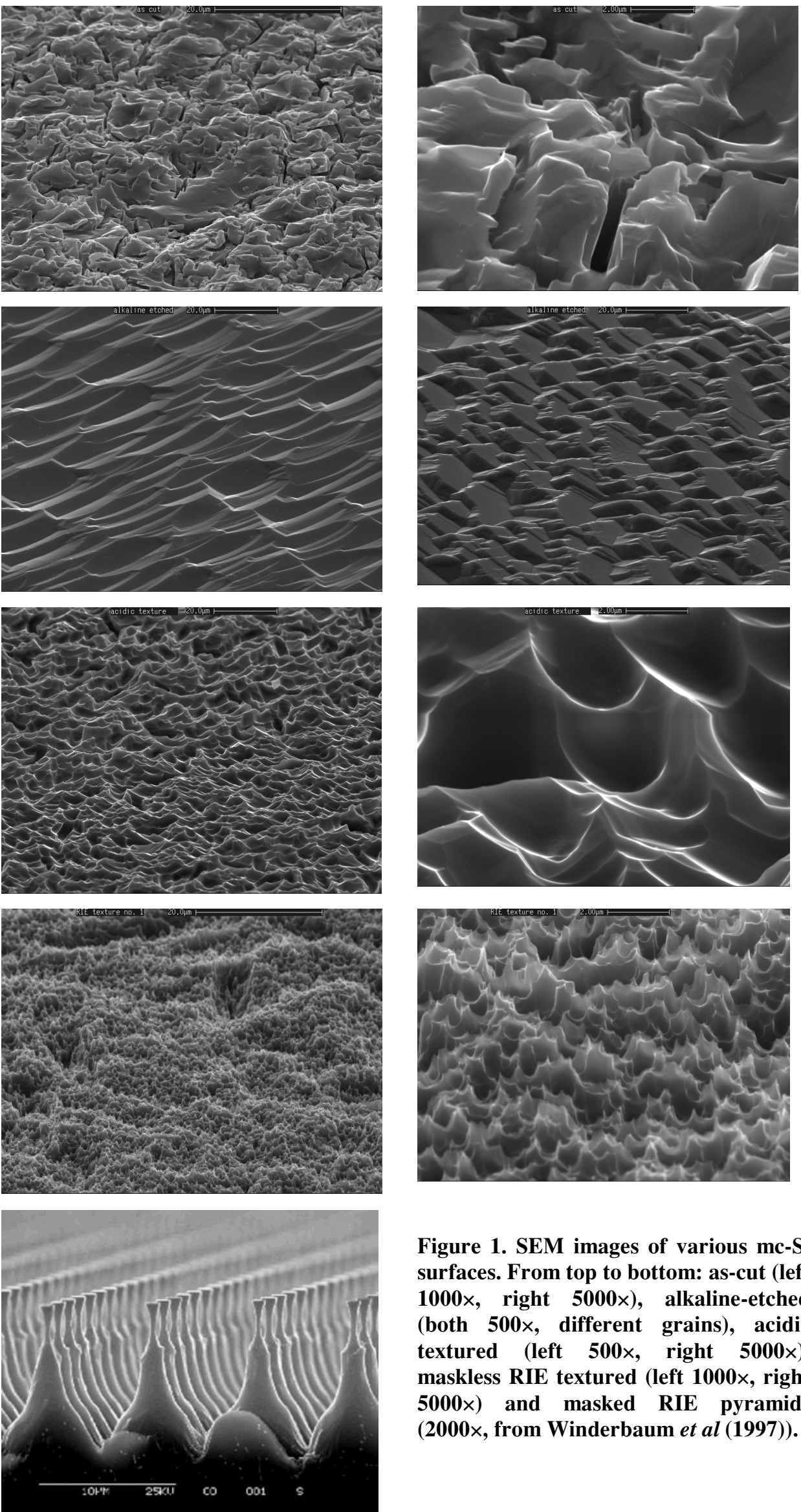

Figure 1. SEM images of various mc-Si surfaces. From top to bottom: as-cut (left $1000 \times$, right $5000 \times)$, alkaline-etched (both $500 \times$, different grains), acidic textured (left $500 \times$, right $5000 \times$ ), maskless RIE textured (left $1000 \times$, right $5000 \times)$ and masked RIE pyramids (2000×, from Winderbaum et al (1997)). 


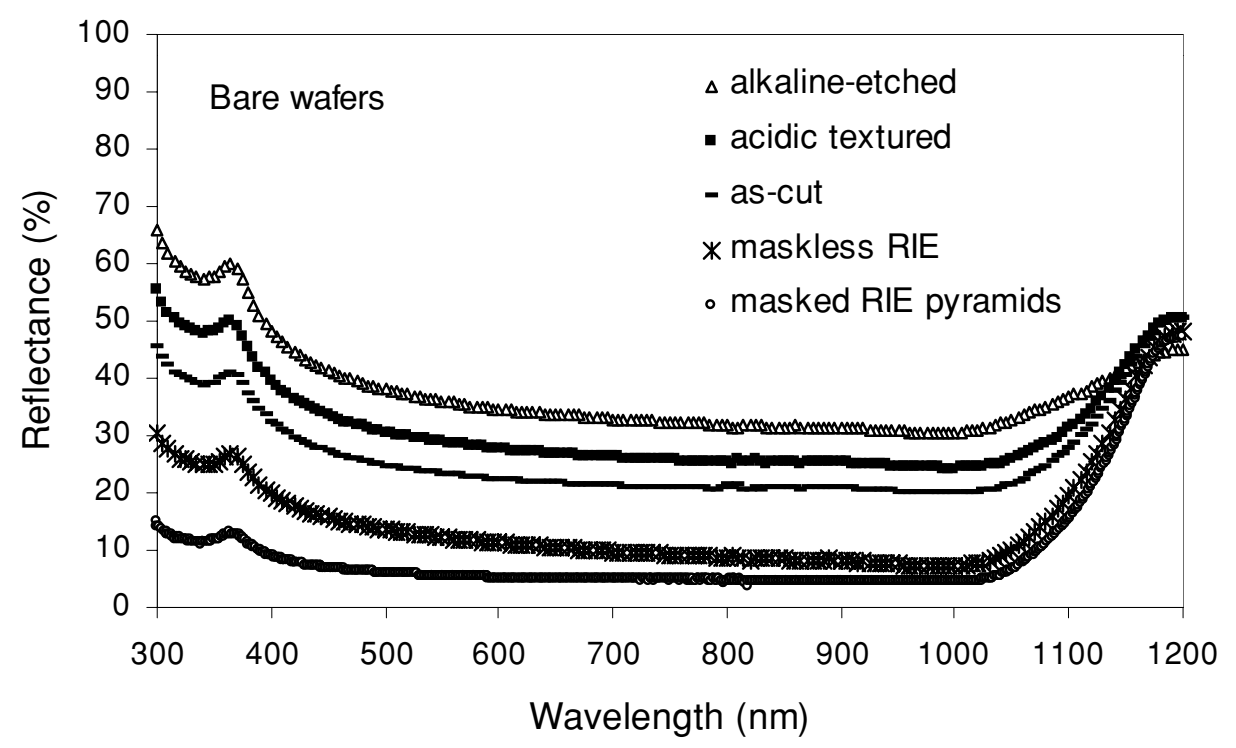

Figure 2. Reflectance measurements of bare alkaline-etched, as-cut, acidic textured, masked RIE and maskless RIE textured multicrystalline silicon wafers.

\subsection{Reflectance Measurements}

Figure 2 shows the results of reflectance measurements on the three types of textured wafers, plus alkaline-etched and as-cut samples. These curves have been used to calculate weighted reflectances for the AM1.5G spectrum. The results are shown in Table 1.

\begin{tabular}{|l|l|l|l|c|c|}
\hline & As-cut & $\begin{array}{l}\text { Alkaline } \\
\text { etched }\end{array}$ & $\begin{array}{l}\text { Acidic } \\
\text { textured }\end{array}$ & $\begin{array}{l}\text { Maskless } \\
\text { RIE }\end{array}$ & $\begin{array}{l}\text { Masked RIE } \\
\text { pyramids }\end{array}$ \\
\hline Bare & $22.6 \%$ & 34.4 & 27.6 & 11.0 & $5.6^{*}$ \\
\hline With SiN AR coating & 7.6 & 9.0 & 8.0 & 3.9 & n/a \\
\hline SiN \& encapsulated & 8.9 & 12.9 & 9.2 & 7.6 & n/a \\
\hline
\end{tabular}

Table 1. Weighted AM1.5 reflectivities of bare, SiN-coated and SiN-coated/encapsulated wafers with various surfaces. Data marked * from Winderbaum et al. 1997.

As discussed above, the alkaline etch, which is optimised for removing the saw damage, not for texturing, produces a high reflectance. In fact, for samples of this thickness (about $275 \mu \mathrm{m}$ ), a perfectly polished surface would also produce a reflectance of around $34 \%$. The as-cut surface exhibits reasonable reflection control, but, as shown below, is electronically unacceptable for cell production.

The acidic textured sample produced a weighted reflectance somewhat lower than the alkaline etch, but certainly not as good as the RIE textured samples. However, this may be offset by the relative ease of implementing acidic texturing. Purely in terms of reflectance though, the results for the RIE texturing processes are very good. It should be noted that the reflectance of acidic textured wafers could be improved with further optimisation, as shown by De Wolf et al, 2000, who have achieved weighted reflectances on bare wafers of about 22\% (as opposed to $27.6 \%$ here).

While the results of the bare wafers give a good indication of the effectiveness of the various texturing methods, in a real cell the surface will have an encapsulated antireflection coating (ARC), which will further change the overall reflection properties. Therefore, we have measured the reflectance of the textured surfaces coated with a plasma-enhanced chemical vapour deposited (PECVD) SiN film, both before and after encapsulation. A major advantage of PECVD SiN films is that they also electronically passivate the surface, leading to reduced recombination losses. Hence there are two separate issues to consider here: firstly, the optical properties of the $\mathrm{SiN}$ coated textured surfaces, and secondly, how well the SiN film passivates the textured surfaces. The quality of the surface passivation is revealed by lifetime measurements, which are presented in the next section. Firstly though, we present reflectance results on SiN coated wafers. 


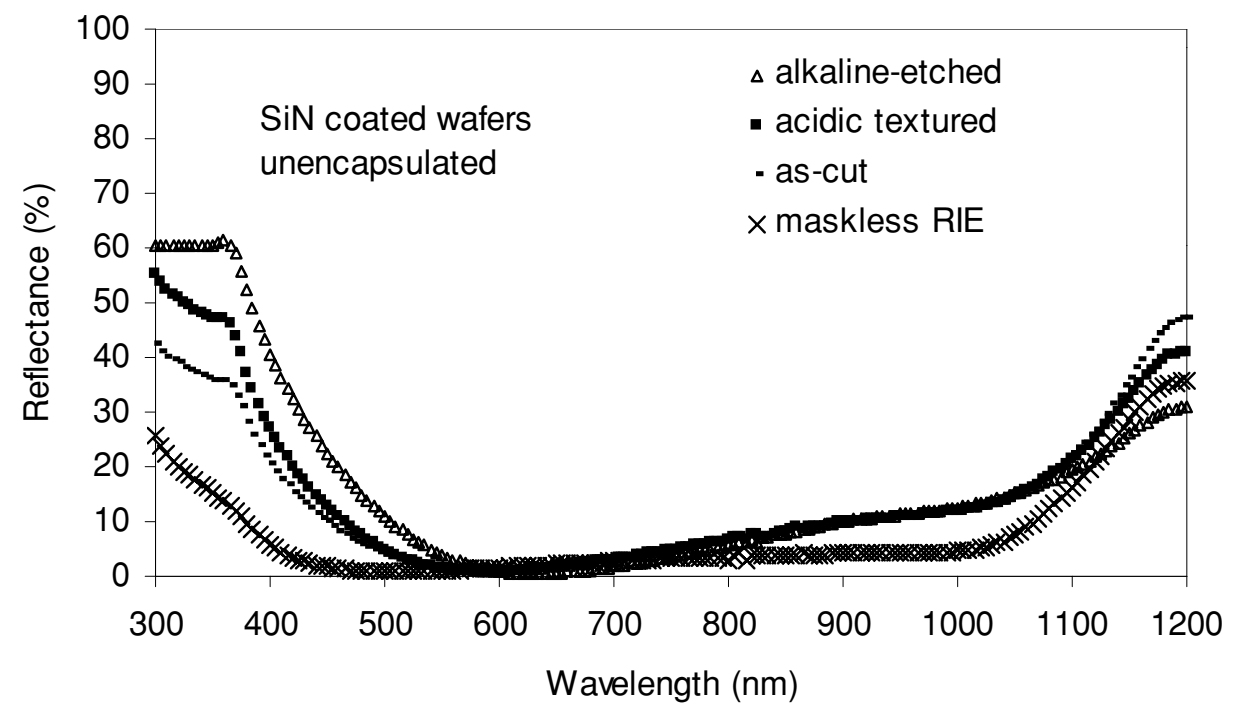

Figure 3. Reflectance measurements of SiN coated, but unencapsulated, multicrystalline silicon wafers.

The results before encapsulation are shown in Figure 3. As expected, all have dramatically reduced reflectance in comparison to the bare wafers, as also indicated by the weighted reflectances in Table 1 .

The results for the unencapsulated wafers show that the difference between the various textures and the alkaline etched surface has been reduced. Initially, this appears to diminish the relative benefit of the texturing processes. However, this does not accurately represent the real case of a finished solar cell inside a module. When encapsulated under glass, and with a rear reflector in place, the samples with more textured surfaces will have relatively improved reflectances due to light-trapping. This is indicated in the third row of Table 1 . These encapsulated measurements were taken with a $90 \%$ reflective mirror behind the wafer. This reasonably closely simulates the case in a finished cell which has a rear reflectivity of about $70 \%$ due to the Al back surface field.

A more direct illustration of the light-trapping effect is given by the infra-red region of the reflectance curves of Figure 4. At wavelengths greater than $1000 \mathrm{~nm}$, the benefits of light-trapping in the textured wafers as opposed to the alkaline-etched sample is clear. The RIE textured wafer in particular exhibits good light-trapping. The reflectivity curves shown in the previous figures can not be interpreted in this way, since no mirror at the rear was used, and there was no planar glass surface at the front. Note that the apparent reduction in reflection in the UV region after encapsulation is caused by absorption within the encapsulating material.

The results in Table 1 certainly show that both the maskless RIE and wet acidic texturing methods give a significant reduction in reflectance in comparison to the alkaline-etched wafer after encapsulation. Comparable results for the masked RIE process were not available, but it is reasonable to assume that they would be lower even that the maskless RIE value of 7.6\%. One important observation is that the difference between the acidic and RIE process has been further reduced after encapsulation. In comparison to the alkaline-etched wafer, the acidic wafer gives a $3.7 \%$ reduction in reflectance, while the maskless RIE wafer yields a gain of $5.3 \%$. It may be that the extra cost of RIE processes is warranted by this extra gain.

\subsection{Lifetime Measurements}

As mentioned above, an important requirement of any texturing regime is that the surface is not too damaged, and can therefore be passivated by the SiN coating. This is necessary to maintain cell voltage, and also to ensure that any benefits of an improved blue response of optimised emitters are realised. It has been shown before that maskless RIE textured surfaces are satisfactory after a damage-removal etch, or even without one in some cases (Damiani et al, 2000). This section is concerned with the surface quality of the wet acidic-textured samples, which can be assessed by lifetime measurements on SiN coated wafers.

The results are shown in Table 2. For a sample that is completely unpassivated, we would expect an effective lifetime of less than $1 \mu \mathrm{s}$ when measuring with white light. This is precisely what occurs for the as-cut wafer, a direct result of the severe surface damage. For a shiny-etched (planar) sample, the effective lifetime was $67 \mu \mathrm{s}$, 


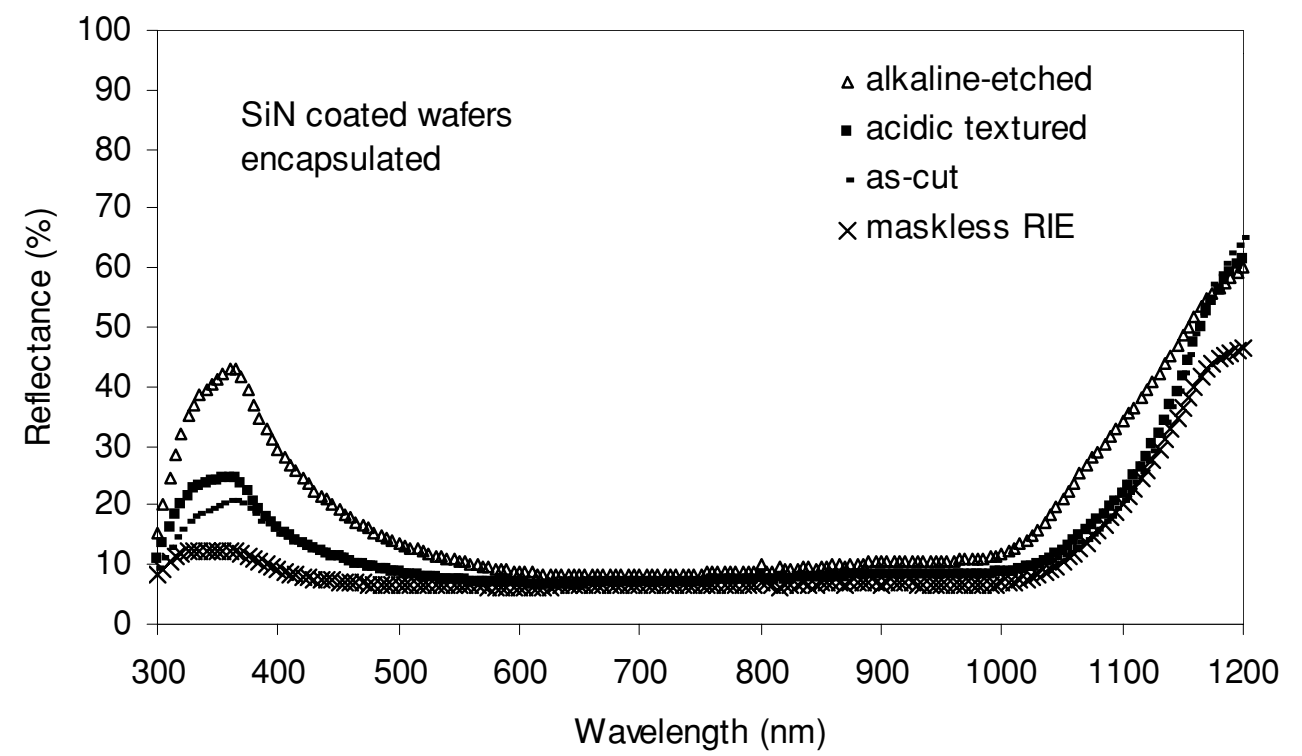

Figure 4. Reflectance measurements of SiN coated and encapsulated, multicrystalline silicon wafers.

compared with $35 \mu$ s for the acidic textured wafer. This reveals that the acidic textured surface can be adequately passivated, although the increased surface area results in a greater amount of surface recombination than occurs in a planar sample.

\begin{tabular}{|l|l|l|}
\hline As-cut & Shiny-etched & Acidic textured \\
\hline $0.8 \mu \mathrm{s}$ & $67 \mu \mathrm{s}$ & $35 \mu \mathrm{s}$ \\
\hline
\end{tabular}

Table 2. Lifetime measurements of SiN coated textured multicrystalline silicon wafers.

\subsection{Cell Results}

A batch of small area $\left(4 \mathrm{~cm}^{2}\right) \mathrm{mc}-\mathrm{Si}$ cells were made at ANU to quantify the improvement in short-circuit current obtained with acidic texturing. They had evaporated Al back-surface fields, SiN passivated emitters and evaporated metal fingers. To provide a basis for comparison, cells with SiN were also fabricated on shiny-etched (planar) wafers. Table 3 lists the short-circuit current density averaged for a number of such cells, which were made using wafers from a standard BPSolar ingot. By averaging the short-circuit current of numerous cells, the uncertainty caused by slightly different SiN thicknesses is reduced, an important factor since film thickness cannot always be precisely replicated with our deposition system.

\begin{tabular}{|l|c|c|c|}
\hline & Shiny-etched (14 cells) & Acidic textured (12 cells) & \% increase \\
\hline Average $\mathrm{J}_{\mathrm{SC}}\left(\mathrm{mAcm}^{-2}\right)$ & $29.3( \pm 0.8)$ & $30.4( \pm 0.9)$ & $3.7 \%$ \\
\hline
\end{tabular}

Table 3. Comparison of average short-circuit current densities (and standard deviations) for shiny-etched and acidic textured mc-Si $4 \mathrm{~cm}^{2}$ cells (unencapsulated) made at the ANU.

The results show that the acidic texturing provides a clear boost to the current, by slightly more than $1 \mathrm{mAcm}{ }^{-2}$, or almost $4 \%$, on average. The reflectivity measurements above indeed showed that a current increase should result from the acidic texturing, but based on reflectance alone, this increase should have only been around $1 \%$. This figure represents the difference between the reflectivity of acidic etched and alkaline etched samples, which are optically similar to shiny-etched wafers. The extra gain in current observed here most likely results from the oblique coupling of light into the cell, resulting in generation closer to the junction, and therefore a greater probability of collection. Similar studies on maskless RIE textured wafers (Damiani $e$ t al, 2000) produced an even greater increase in current in comparison to planar controls, from 28.25 to $30.63 \mathrm{mAcm}^{-2}$ (an $8.4 \%$ gain).

It would be expected that this relative improvement in current would become even greater after encapsulation, because of the light-trapping properties of the acidic-textured surface. However, due to reflection from the top of 
the glass (about 4.5\%), the magnitude of the current will in fact decrease after encapsulation for both acidic textured and planar cells, but the decrease should be less for textured cells.

Table 4 shows that this is indeed the case. It gives a comparison of the short-circuit currents of two individual mc-Si cells, one of which was acidic textured and the other shiny-etched, before and after encapsulation. The results show that the gain from acidic texturing increased from 3.3\% to 5.1\% after encapsulation (an increase of $1.8 \%$ ). This is similar to the results of De Wolf et al., 2000, who observed an improvement of $6.4 \%$ for a module made of acidic textured cells in comparison to a module of alkaline-etched cells. The results from Table 1 indicate that the difference in reflectance between planar and acidic textured wafers widens from $1 \%$ to $3.7 \%$ after encapsulation. This suggests that a further $2.7 \%$ relative current gain can reasonably be expected after encapsulation of acidic textured samples compared with planar cells, a little higher but still in reasonable agreement with the observed increase of $1.8 \%$. Generally speaking, these results certainly show that acidic texturing provides significant gains in terms of current in BPSolar material.

\begin{tabular}{|l|c|c|c|}
\hline Cell & 91DA shiny-etched & 90AB acidic textured & \% difference \\
\hline $\mathrm{J}_{\mathrm{SC}}$ before encapsulation & 30.7 & 31.7 & $3.3 \%$ \\
$\mathrm{~J}_{\mathrm{SC}}$ after encapsulation & 29.7 & 31.2 & $5.1 \%$ \\
\% change in $\mathrm{J}_{\mathrm{SC}}$ & $-3.4 \%$ & $-1.6 \%$ & $1.8 \%$ \\
\hline
\end{tabular}

Table 4. Comparison of short-circuit current densities for an acidic textured and a shiny-etched $\mathrm{SiN}$ coated $\mathrm{mc}-\mathrm{Si} \mathbf{4 \mathrm { cm } ^ { 2 }}$ cell before and after encapsulation.

\section{CONCLUSIONS}

All three texturing methods examined - wet acidic texturing, and masked and maskless RIE texturing significantly reduce reflection losses in solar cells. The reduction in reflection is greatest for masked RIE pyramids, followed by maskless RIE, and then acidic texturing, and this is likely to also be the order of greatest improvement in cell performance. However, the relative difference between the methods is substantially reduced after antireflection-coating and encapsulation. Also, the cost of implementation is likely to be substantially less for acidic texturing then for either RIE process, especially masked RIE. It is therefore difficult to accurately quantify, at this stage, which technique offers the best prospects for commercial implementation.

\section{ACKNOWLEDGMENTS}

This work has been supported by the Australian Greenhouse Office through the Renewable Energy Commercialisation Program (RECP), and by the New South Wales Ministry of Energy through the Sustainable Energy Research and Development Fund (SERDF). The authors are grateful to James Cotsell and Mick Stuckings from CSES at the ANU for assisting with encapsulation and reflectance measurements.

\section{REFERENCES}

Damiani B., Ludemann R., Ruby D., Zaidi S. and Rohatgi A. (2000), Development of RIE-textured silicon solar cells, in Proceedings of the 28th IEEE Photovoltaic Specialists Conference, Anchorage, Alaska, 2000.

De Wolf S., Choulat P., Vazsonyi E., Einhaus R., Van Kerschaver E., De Clercq K and Szlufcik J. (2000), Towards industrial application of isotropic texturing for multi-crystalline silicon solar cells, in Proceedings of the 16th European Photovoltaic Solar Energy Conference, Glasgow, U.K., 2000, pp. 1521-1523.

Ruby D., Zaidi S., Roy M. and Narayanan M. (1999), Plasma texturing of silicon solar cells, in Proceedings of the $9^{\text {th }}$ Workshop on the Role of Impurities and Defects in Silicon Device Processing, Colorado, USA, 1999, pp. 179182.

Winderbaum S., Reinhold O. and Yun F. (1997), Reactive ion etching (RIE) as a method for texturing polycrystalline silicon solar cells, Solar Energy Materials and Solar Cells, 46, 239-248. 